OPEN ACCESS

Edited by:

Angel Medina,

Cranfield University, United Kingdom

Reviewed by:

Esther Garcia-Cela,

Cranfield University, United Kingdom

Jéssica Gil-Serna

Complutense University of Madrid,

Spain

Carol Verheecke

Université Paul Sabatier, France

*Correspondence:

Gary L. Windham

gary.windham@ars.usda.gov

Specialty section:

This article was submitted to Fungi and Their Interactions,

a section of the journal

Frontiers in Microbiology

Received: 31 July 2017

Accepted: 10 April 2018

Published: 24 April 2018

Citation:

Windham GL, Williams WP,

Mylroie JE, Reid CX and

Womack ED (2018) A Histological

Study of Aspergillus flavus

Colonization of Wound Inoculated

Maize Kernels of Resistant

and Susceptible Maize Hybrids

in the Field. Front. Microbiol. 9:799.

doi: 10.3389/fmicb.2018.00799

\section{A Histological Study of Aspergillus flavus Colonization of Wound Inoculated Maize Kernels of Resistant and Susceptible Maize Hybrids in the Field}

\author{
Gary L. Windham ${ }^{1 *}$, William P. Williams ${ }^{1}$, J. E. Mylroie' ${ }^{1}$, Cedric X. Reid ${ }^{2}$ and \\ Erika D. Womack
}

${ }^{1}$ Corn Host Plant Resistance Research Unit, United States Department of Agriculture-Agricultural Research Service, Mississippi State, MS, United States, ${ }^{2}$ Department of Biochemistry, Molecular Biology, Entomology, and Plant Pathology, Mississippi State University, Mississippi State, MS, United States

Aspergillus flavus colonization in developing kernels of maize single-cross hybrids resistant (Mp313E $\times$ Mp717) and susceptible (GA209 $\times$ T173) to aflatoxin accumulation was determined in the field over three growing seasons (2012-2014). Plants were hand pollinated, and individual kernels were inoculated with a needle dipped in a suspension of $A$. flavus conidia 21 days after pollination. Kernels were harvested at 1 - to 2-day intervals from 1 to 21 days after inoculation (DAl). Kernels were placed in FAA fixative, dehydrated, embedded in paraffin, sectioned, and stained with toluidine blue. Kernels were also collected additional kernels for aflatoxin analyses in 2013 and 2014. At 2 DAl, A. flavus hyphae were observed among endosperm cells in the susceptible hybrid, but colonization of the endosperm in the resistant hybrid was limited to the wound site of the resistant hybrid. Sections of the scutellum of the susceptible hybrid were colonized by $A$. flavus by 5 DAl. Fungal growth was slower in the resistant hybrid compared to the susceptible hybrid. By 10 DAl, A. flavus had colonized a large section of the embryo in the susceptible hybrid; whereas in the resistant hybrid, approximately half of the endosperm had been colonized and very few cells in the embryo were colonized. Fungal colonization in some of the kernels of the resistant hybrid was slowed in the aleurone layer or at the endosperm-scutellum interface. In wounded kernels with intact aleurone layers, the fungus spread around the kernel between the pericarp and aleurone layer with minimal colonization of the endosperm. Aflatoxin $\mathrm{B}_{1}$ was first detected in susceptible kernel tissues 8 DAl in $2013(14 \mu \mathrm{g} / \mathrm{kg})$ and 2014 (18 $\mu \mathrm{g} / \mathrm{kg})$. The resistant hybrid had significantly lower levels of aflatoxin accumulation compared to the susceptible hybrid at harvests 10, 21, and 28 DAl in 2013, and 20 and 24 DAl in 2014. Our study found differential $A$. flavus colonization of susceptible and resistant kernel tissues, and that the aleurone and the outer layer of the scutellum slowed the rate of colonization by $A$. flavus.

Keywords: Aspergillus flavus, corn (Zea mays), maize, aflatoxins, histology, pathogenesis, resistance 


\section{INTRODUCTION}

Preharvest infection of maize (Zea mays L.) kernels by Aspergillus flavus Link:Fr and subsequent accumulation of aflatoxin is a chronic economic and food safety problem in the southeastern United States and is a sporadic problem in the midwestern United States corn belt (Payne, 1992; Park and Liang, 1993; Widstrom, 1996; Wu, 2015). It is estimated that aflatoxins cause an annual loss of $\$ 163$ million to maize producers in the United States ( $\mathrm{Wu}, 2015)$. Aflatoxin contaminations of maize is also a problem for maize growers in Asia, sub-Saharan Africa, and in Europe (Darwish et al., 2014; Wu, 2015; Battilani et al., 2016; Agbetiameh et al., 2018). Aflatoxin contamination as a preharvest problem in maize was first reported in 1975 and 1976 (Anderson et al., 1975; Lillehoj et al., 1975). Aflatoxin is one of the most potent carcinogens produced in nature and is commonly found in maize kernels infected with A. flavus (Castegnaro and McGregor, 1998). Consumption of aflatoxin-contaminated foods is a major cause of liver toxicity and hepatocellular carcinoma, the fifth most common cancer in the world (Wild and Hall, 2000; Wang et al., 2001; Ayeni, 2015). It has been estimated that five billion people worldwide are exposed to dietary aflatoxin on a regular basis (Wu, 2015). Individuals exposed to both high levels of aflatoxin and chronic hepatitis B virus infection have an increased risk of liver cancer (Liu and $\mathrm{Wu}, 2010$ ). The U.S. Food and Drug Administration limits the sale of grain with aflatoxin levels exceeding $20 \mu \mathrm{g} / \mathrm{kg}$. Grain exceeding $20 \mu \mathrm{g} / \mathrm{kg}$ cannot be shipped across state lines and can only be used for livestock feed. Aflatoxin contaminated grain not exceeding 100,200 , or $300 \mu \mathrm{g} / \mathrm{kg}$ can be used as feed for breeding beef cattle and swine, finishing swine, or finishing beef cattle in the United States, respectively. Globally, over 100 nations worldwide have established maximum tolerable levels for aflatoxin in food ranging from 2 to $10 \mu \mathrm{g} / \mathrm{kg}(\mathrm{Wu}$, 2015).

Plant resistance is considered to be one of the most desirable strategies for managing aflatoxin accumulation in maize. The development of aflatoxin resistant germplasm has been the primary emphasis of a number of research organizations, particularly the United States Department of Agriculture-Agricultural Research Service (USDA-ARS) (Scott and Zummo, 1990, 1992; Williams and Windham, 2001, 2006; Williams et al., 2015). The USDA-ARS Corn Host Plant Resistance Research Unit located at Mississippi State, MS, United States, released the first two maize lines (Mp313E, Mp420) with resistance to kernel infection by A. flavus (Scott and Zummo, 1990, 1992). More recently, four additional germplasm lines (Mp715, Mp717, Mp718, Mp719) with resistance to aflatoxin accumulation have been released (Williams and Windham, 2001, 2006, 2012). Progress has been made in identifying molecular markers associated with resistance to aflatoxin accumulation in our lines (Brooks et al., 2005; Warburton et al., 2009, 2011). Studies have also been conducted to determine cell constituents and metabolic pathways associated with A. flavus resistance in maize (Hawkins et al., 2015; Tang et al., 2015). However, little information is available on physical barriers inside the kernels that contribute to host resistance mechanisms in these germplasm.
The objective of our study were to compare colonization of $A$. flavus in maize resistant and susceptible to aflatoxin accumulation in order to identify sites within developing kernels that are associated with limiting fungal spread.

\section{MATERIALS AND METHODS}

\section{Fungal Strain and Inoculum Production}

Aspergillus flavus strain NRRL 3357, which is known to produce aflatoxin in maize grain (Windham and Williams, 1998, 2002), was used as inoculum. Cultures were started each spring using freeze-dried plugs supplied by the USDA-ARS Bacterial Food-borne Pathogen and Mycology Research Unit (Peoria, IL, United States). Inoculum was increased on sterile corn cob grits (Grit-O-Cobs ${ }^{\circledR}$, The Andersons Inc., Maumee, OH, United States) in 500-ml flasks, each containing $50 \mathrm{~g}$ of grits and $100 \mathrm{ml}$ of sterile, distilled water, and incubated at $28^{\circ} \mathrm{C}$ for 3 weeks. Conidia in each flask were washed from the grits using $500 \mathrm{ml}$ sterile distilled water containing 20 drops of Tween 20 (Sigma-Aldrich, St. Louis, MO, United States, Cat. \# P1379) per liter and filtered through four layers of sterile cheesecloth. Inoculum not used immediately was refrigerated at $4^{\circ} \mathrm{C}$. Fresh A. flavus inoculum was collected each week.

\section{Maize Kernel Production, Inoculation, and Harvest}

An A. flavus resistant single-cross maize hybrid $(\mathrm{Mp} 313 \mathrm{E} \times \mathrm{Mp} 717)$ and a susceptible single-cross maize hybrid (GA209 × T173) were grown at the R. R. Foil Plant Science Research Center located at Mississippi State University, MS, United States, in 2012-2014. The inbred parents (Mp313E and Mp717) of the resistant hybrid typically have lower levels of aflatoxin accumulation and A. flavus colonization when compared with inbred parents (GA209 and T173) of the susceptible hybrid in field trials (Scott and Zummo, 1990; Williams and Windham, 2006; Brown et al., 2016). Plants were hand pollinated and covered with a paper pollination bag (Lawson 402, Northfield, IL, United States). Individual kernels were inoculated 21 days after pollination with a size 12 quilting needle (Entaco Limited, Worcestershire, England, Cat.\# JJ12012) imbedded in a pencil eraser with $1 \mathrm{~mm}$ of the pointed tip exposed (Figure 1A). A scalpel was used to make a $2.5 \mathrm{~cm}$ cut across the ear husks, and then two longitudinal cuts $5.0 \mathrm{~cm}$ in length were made. The husks were peeled back to expose the developing kernels. Individual kernels were inoculated by dipping the needle tip in a suspension of $A$. flavus conidia $\left(1 \times 10^{8}\right.$ conidia $\left./ \mathrm{ml}\right)$ and inserting the needle into the center of the exposed crown of the kernel. Husks were repositioned over the kernels, secured with a rubber band, and the ear was covered with a clear plastic bag. To examine the potential for A. flavus movement into adjacent, uninoculated kernels, eight kernels surrounding a selected kernel were inoculated with A. flavus as described above. Developing kernels are attached to the cob in paired rows and share vascular tissues that connect to the main vascular bundle in the cob (Smart et al., 1990). By inoculating kernels in rows adjacent to the uninoculated 

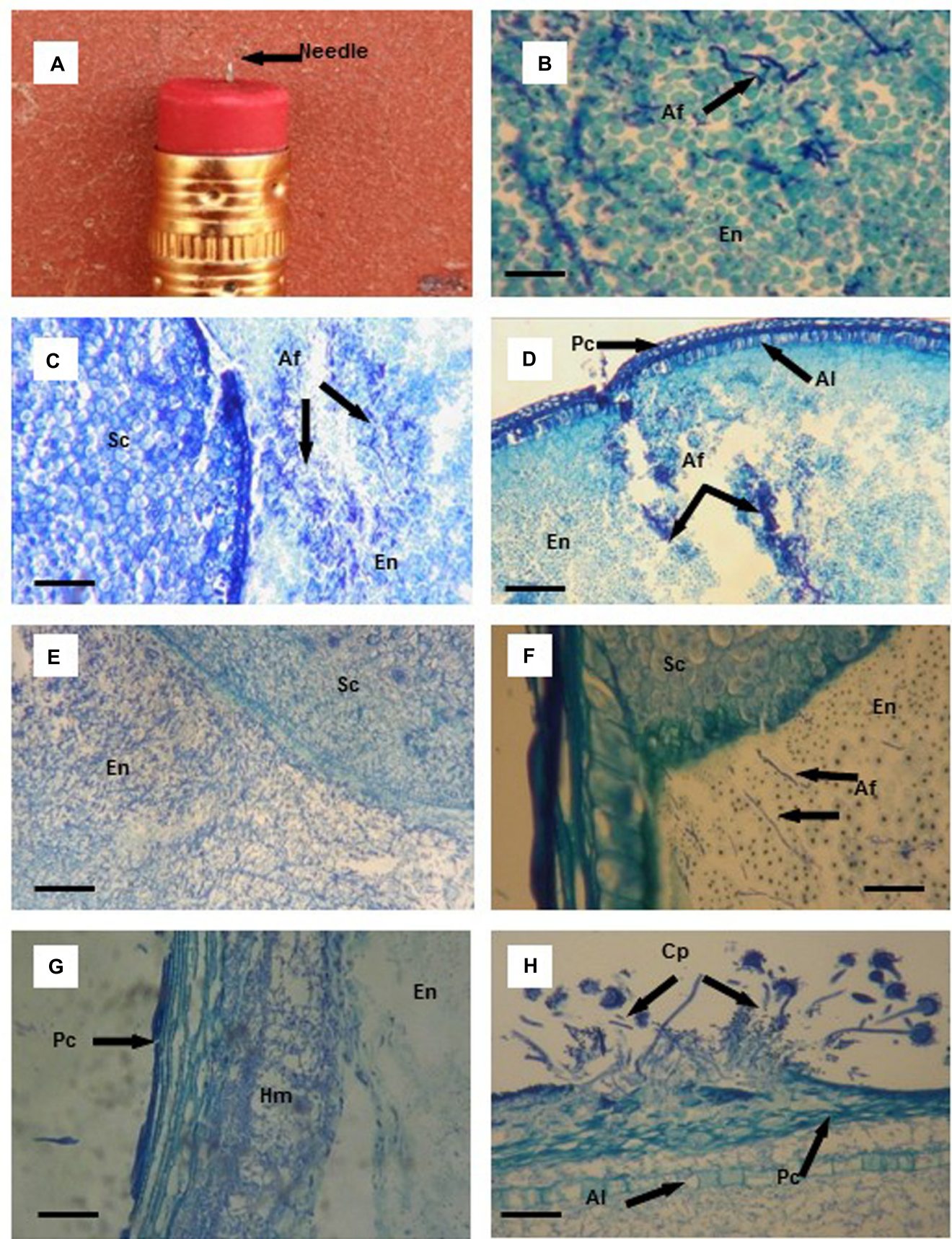

FIGURE 1 | Thin tissue sections (B-H) from aflatoxin resistant (Mp313E $\times$ Mp717) and susceptible (Ga209 $\times$ T173) maize kernels infected with Aspergillus flavus (Af) using a needle (A) imbedded in a pencil eraser as the artificial inoculation device. (B) Initial colonization of endosperm (En) tissues by A. flavus hyphae (black arrows). (C) Colonization of endosperm (En) tissue of the susceptible hybrid by A. flavus 3 days after inoculation (DAl); no fungal colonization of the scutellum (Sc). (D) A. flavus hyphae concentrated at the wound of the endosperm (En), aleurone layer (Al), and pericarp (Pc) in the resistance hybrid 3 DAl. (E) Cells containing starch granules disappear with extensive colonization of the endosperm (En) and scutellum (Sc) of the susceptible hybrid by A. flavus 5 DAl. (F) A. flavus hyphae advance toward the scutellum (Sc) through the endosperm (En) in the resistant hybrid. (G) A. flavus hyphal mat ( $\mathrm{Hm}$ ) formed between the pericarp (Pc) and the outer layer of the endosperm (En) 6 DAl. (H) A. flavus conidiophores (Cp) found on the kernel surface after the fungus had erupted through the pericarp (Pc) 7 DAl; extensive A. flavus colonization between the pericarp and aleurone layer (Al), and in the endosperm. Scale bars: (B) $30 \mu \mathrm{m} ;$ (F) $50 \mu \mathrm{m} ;(\mathbf{G}, \mathbf{H}) 100 \mu \mathrm{m} ;(\mathbf{C}-\mathbf{E}) 200 \mu \mathrm{m}$.

kernels, it would allow us to determine if it was possible for A. flavus to colonize adjacent, uninoculated kernels via the vascular tissue of the cob. For histological studies, three ears of each hybrid were harvested at 1- to 2-day intervals from 1 to 21 days after inoculation (DAI). Four to five inoculated kernels from each ear were placed in vials containing the fixative FAA 
(formalin-acetic acid-alcohol) (Berlyn and Miksche, 1976) and kept in a refrigerator at $4^{\circ} \mathrm{C}$.

\section{Histological Methods}

Kernels were removed from the FAA fixative, dehydrated through a graded tertiary butyl alcohol series, and embedded in Para last Plus wax (Sigma-Aldrich, St. Louis, MO, United States, Cat. \#P3683) (Berlyn and Miksche, 1976). Tissue sections (10 $\mu \mathrm{m}$ ) were cut longitudinally from the wax-embedded kernels using an American Optical Rotary microtome, affixed to glass microscope slides with Haupt's gelatin adhesive, and stained with $0.05 \%$ aqueous toluidine blue. Cover slips were cemented over stained sections using a Permount mounting media (Fisher Scientific, Fair Lawn, NJ, United States, Cat. \#SP15-100), and viewed with an Olympus BH-2 compound microscope.

\section{Aflatoxin Quantification}

In 2013 and 2014, additional kernels for aflatoxin analyses (four to five from each ear) were collected from the ears harvested for the histological study. Aflatoxin $B_{1}$ is the most common aflatoxin produced by aflatoxin producing Aspergillus species (Mehl and Cotty, 2010) and was the only aflatoxin quantified. In 2013, wound-inoculated and uninoculated kernel samples were collected 2, 4, 6, 8, 9, 10,12, 14, 21, and 28 DAI and prepared for aflatoxin analyses using methodology described by Reid et al. (2016). Briefly, kernels were flash frozen in liquid nitrogen and ground into a fine powder with a mortar and pestle. Ground samples $(200 \mathrm{mg})$ were placed in $2.0 \mathrm{ml}$ micro-centrifuge tubes and $1.0 \mathrm{ml}$ of $(70 / 30, \mathrm{v} / \mathrm{v})$ methanol/water was added to each tube and vortexed for $1 \mathrm{~min}$. Tubes were centrifuged at 14,000 rpm for $5 \mathrm{~min}$. The supernatant was collected and passed through polytetrafluoroethylene syringe filters $(0.45 \mu \mathrm{m})$. The liquid extracts were transferred to auto-sampler vials and aflatoxin $B_{1}$ was quantified using an Agilent 6460 Triple Quadruple tandem mass spectrometer (Reid et al., 2016). Recovery of aflatoxin $B_{1}$ in spiked samples was $90 \%$.

In 2014, kernel samples collected daily 3-10 DAI, and then every other day from 12 to 24 DAI. Kernels were flash frozen in liquid nitrogen and ground with a 2000 Geno/Grinder (SPEX Certiprep, Metuchen, NJ, United States) for $3 \mathrm{~min}$ at 1200 strokes/min. Aflatoxin was extracted from the ground samples and the supernatant treated as described above. Samples were analyzed for aflatoxin $B_{1}$ on an Agilent 1100 Infinity Liquid Chromatography (HPLC) system coupled to fluorescence detector (excitation $362 \mathrm{~nm}$, emission $455 \mathrm{~nm}$ ). Analysis was performed by injecting $10 \mu \mathrm{L}$ of the sample onto a Zorbax Eclipse XDB-C18 $4.6 \mathrm{~mm} \times 150 \mathrm{~mm}, 3.5 \mu \mathrm{m}$ column from Agilent (Santa Clara, CA, United States) with a column temperature of $25^{\circ} \mathrm{C}$. The mobile phase consisted of HPLC grade water, methanol, and acetonitrile (50:40:10 v/v/v) and was pumped at a flow rate of $0.8 \mathrm{ml} / \mathrm{min}$ under isocratic conditions for a total run time of $10 \mathrm{~min}$. Linearity was evaluated with a matrixmatched 10-point calibration curve (replicated twice) ranging from 3.9 to $2,000 \mu \mathrm{g} / \mathrm{kg}$ aflatoxin $\mathrm{B}_{1}$. The least square method was used to estimate the linear regression. A satisfactory linear correlation was determined by the coefficient of determination $\left(R^{2}\right)$. Accuracy of the extraction method was evaluated by performing recovery experiments using ground samples of uninoculated corn kernels spiked with $50 \mu \mathrm{g} / \mathrm{kg}$ aflatoxin $\mathrm{B}_{1}$ $(n=3)$. Recovery of aflatoxin $\mathrm{B}_{1}$ in spiked samples was $93 \%$.

\section{Statistical Analyses of Aflatoxin Data}

All statistical analyses were conducted using the SAS software package (version 9.4; SAS Institute Inc., Cary, NC, United States). Means for aflatoxin concentration were transformed $[\ln (y+1)]$ before statistical analyses. Transformed data was analyzed using the Proc GLM procedure to compare aflatoxin contamination of the resistant and susceptible hybrids for individual harvest dates.

\section{RESULTS}

One advantage our study has over similar studies is that the USDA-ARS maize breeding program has developed aflatoxin resistant maize genotypes through conventional breeding methods. These resistant genotypes can be compared with susceptible genotypes to determine the physical, chemical, and genetic factors that affect $A$. flavus growth and subsequent aflatoxin production in developing kernels. In our present study, we found differences in A. flavus growth and aflatoxin accumulation in the inoculated kernels of the resistant and susceptible maize hybrids.

\section{Colonization of Kernels by A. flavus}

Toluidine blue stained A. flavus hyphae (Figure 1B) a dark blue, and provided excellent contrast between the fungal hyphae and kernel tissues in the endosperm, scutellum, and germ. The infection processes described in Figures $\mathbf{1 C}-\mathbf{H}$ and $\mathbf{2 A - E}$ summarize A. flavus colonization observed in 2012 and 2013. Unseasonably cooler weather (mean ambient temperatures were $4^{\circ} \mathrm{C}$ lower) during early stages of kernel infection in 2014 delayed kernel colonization compared to fungal spread observed in 2012 and 2013. In general, A. flavus initially colonized the starchy endosperm and the area between the pericarp and aleurone in the wound inoculated kernels. Thus, the fungus began a two-pronged approach attacking the scutellum first and finally the germ.

No A. flavus hyphae were observed in kernel tissues $24 \mathrm{~h}$ after inoculation in either of the maize hybrids. By two DAI, small clumps of hyphae were found in the endosperm of the susceptible hybrid and within the wounded area in the endosperm of the resistant hybrid. A. flavus had colonized areas of the endosperm away from the infection site in the susceptible hybrid 3 DAI (Figure 1C); however, hyphae remained concentrated near the wound damaged area in the endosperm of the resistant hybrid (Figure 1D). In the susceptible hybrid 5 DAI, there was extensive fungal colonization of the endosperm and the scutellum (Figure 1E). Starch granules in the endosperm began to disappear as the amount of mycelial growth increased. In the resistant hybrid $5 \mathrm{DAI}, A$. flavus had colonized a smaller amount of the endosperm compared to the area colonized in the susceptible hybrid, and had not invaded the scutellum (Figure 1F). Endosperm cells containing starch granules were still intact. 

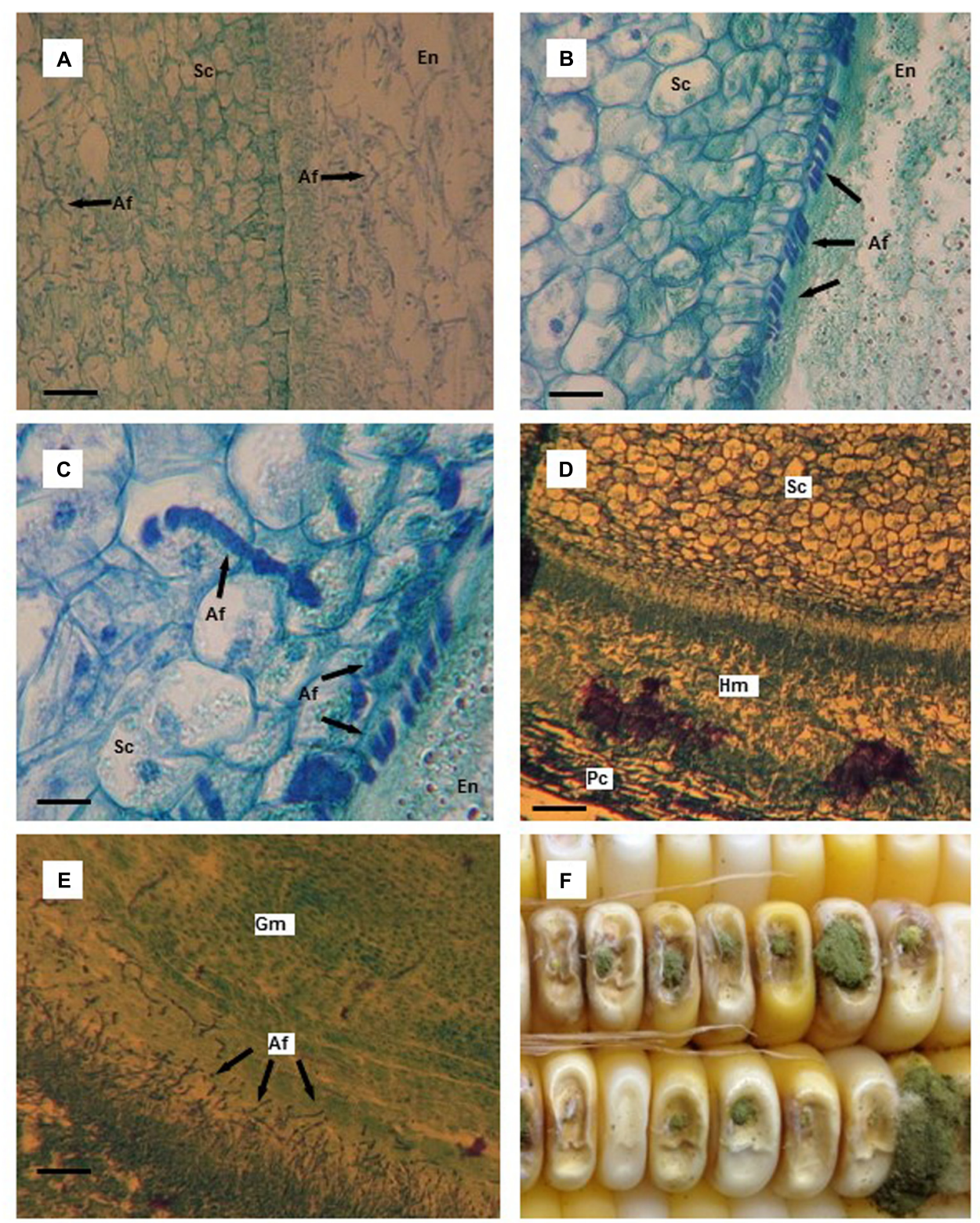

FIGURE 2 | Thin tissue sections (A-E) and whole kernels (F) of aflatoxin susceptible and resistant maize hybrids colonized by Aspergillus flavus. (A) A. flavus colonized endosperm (En) and scutellum (Sc) of the susceptible hybrid 10 days after infection (DAl); starch granules in the endosperm have disappeared and are replaced by mycelium. (B) A. flavus hyphae massed at the endosperm (En)-scutellum (Sc) interface in the resistant hybrid 10 DAl; notice the outer, organized layer of cells in the scutellum. (C) A. flavus penetration of scutellum (Sc) cells in the resistant hybrid 10 DAl. (D) A. flavus hyphal mat found between the pericarp (Pc) and the aleurone layer adjacent to the scutellum (Sc) 12 DAl in the susceptible hybrid. (E) A. flavus invasion of germ (Gm) tissue of the susceptible hybrid 12 DAl. (F) Typical A. flavus growth and sporulation on needle-inoculated kernels. Scale bars: (C) $25 \mu \mathrm{m}$; (B) $50 \mu \mathrm{m}$; (D) $75 \mu \mathrm{m}$; (A,E) $100 \mu \mathrm{m}$.

Our needle inoculator wounded the kernel through the pericarp and the aleurone; the fungus was provided with an entrance to invade the space between these two outer layers of the seed. As the fungus spread between these two layers, a hyphal mass formed separating the pericarp and the aleurone layer (Figure 1G). This invasion route provided the fungus with access to other areas of the developing seed. It was not uncommon for the aleurone layer to remain intact as A. flavus spread between the two layers. In one wound-inoculated kernel of the susceptible hybrid, the pericarp was penetrated by the needle inoculator, but the aleurone layer was not broken. A. flavus spread $95 \%$ of the way around the seed by 6 DAI solely by moving between the pericarp and aleurone layer. As A. flavus hyphae spread around the kernel, only a very small area of the endosperm and scutellum were invaded by the fungus.

Sporulation of A. flavus was visible on the outer surface of the wound-inoculated kernels beginning 4-5 DAI. Typically, the first conidiophores observed were produced by mycelium growing at 
the wound site. But additional areas of conidiophore production were observed externally on the kernel surface when the fungus erupted through the pericarp (Figure $\mathbf{1 H}$ ).

By 10 DAI in the kernel tissue of the susceptible hybrid, mycelium had ramified the endosperm and very few starch granules were visible (Figure 2A). Essentially, the starchy endosperm had been replaced by a mass of A. flavus mycelium. Also, the scutellum was heavily colonized by the fungus. In a resistant kernel $10 \mathrm{DAI}, A$. flavus hyphae had spread through the endosperm and were found concentrated at the endosperm-scutellum interface in a somewhat orderly manner (Figure 2B). The outer layer of the scutellum had limited the amount of infection. Very few areas of the scutellum were infected by the fungus which had only grown 2-4 layers deep into the scutellum. The penetrating hyphae appeared to be constricted as the fungus penetrated cell walls in the scutellum (Figure 2C).

The scutellum in the susceptible hybrid kernels was heavily colonized by 12 DAI via the scutellum-endosperm interface and also by fungal spread around the kernel between the pericarp and aleurone layer. The scutellum was invaded from a hyphal mass that formed between the pericarp and aleurone layer adjacent to the scutellum and opposite the endosperm (Figure 2D). The germ was also infected by A. flavus from the endosperm (Figure 2E). At later harvest dates, the scutellum and the germ in the kernels of the resistant hybrid were invaded by A. flavus. Conidiophore development externally increased through the last harvest dates, but was somewhat less in the resistant hybrid compared to the susceptible hybrid (Figure 2F).

In 2012 and 2013, uninoculated kernels that were paired with A. flavus inoculated kernels (all surrounding kernels had been inoculated to insure the infection of one of the paired kernels) were harvested at 7, 14, and 21 DAI to determine fungal spread via the rachilla (shared vascular tissue). A. flavus was not found internally in any of the uninoculated kernels even though these kernels were surrounded by kernels covered with abundant $A$. flavus mycelia. Fungal growth was also not observed in controlled, uninoculated kernels harvested at various dates during the study.

\section{Aflatoxin Accumulation}

In 2013, aflatoxin was first detected $(14 \mu \mathrm{g} / \mathrm{kg})$ in kernels of the susceptible hybrid 8 DAI (Table 1). Aflatoxin levels in the susceptible hybrid increased each harvest date, with the exception of $12 \mathrm{DAI}$, until the last harvest date (28 DAI). Aflatoxin was only detected in the kernels of the resistant hybrid at one harvest date (14 DAI). Aflatoxin accumulation in the infected kernels at 8 and 10 DAI would have coincided with extensive infection of the endosperm and, in later harvest dates (21 and $28 \mathrm{DAI})$ aflatoxin accumulation would have coincided with infection of the scutellum and germ. Significantly higher levels of aflatoxin contamination were found in the susceptible hybrid than in the resistant hybrid 10, 21, and 28 DAI.

In 2014, aflatoxin was detected in small amounts $(1-8 \mu \mathrm{g} / \mathrm{kg})$ in kernels of the resistant hybrid from 4 to 9 DAI (Table 2). Aflatoxin was first found in kernels of the susceptible hybrid 8 DAI. Aflatoxin accumulation at levels lower than $20 \mu \mathrm{g} / \mathrm{kg}$ were detected in kernels of the susceptible hybrid 8, 9, and 10 DAI.
TABLE 1 | Aflatoxin accumulation in the developing maize kernels of resistant (Mp313E $\times$ Mp717) and a susceptible (GA209 $\times$ T173) hybrids inoculated with Aspergillus flavus in 2013 (21 days after pollination).

\begin{tabular}{lcc}
\hline Days after & \multicolumn{2}{c}{ Aflatoxin $(\boldsymbol{\mu g} / \mathbf{k g})$} \\
\cline { 2 - 3 } Inoculation & GA209 $\times$ T173 & Mp313E $\times$ Mp717 \\
\hline 2 & $0 \pm 0$ & $0 \pm 0$ \\
4 & $0 \pm 0$ & $0 \pm 0$ \\
6 & $0 \pm 0$ & $0 \pm 0$ \\
8 & $14 \pm 24$ & $0 \pm 0$ \\
10 & $83 \pm 71^{*}$ & $0 \pm 0$ \\
12 & $0 \pm 0$ & $0 \pm 0$ \\
14 & $126 \pm 159$ & $125 \pm 216$ \\
21 & $3376 \pm 2631^{*}$ & $0 \pm 0$ \\
28 & $1370 \pm 583^{*}$ & $0 \pm 0$ \\
\hline
\end{tabular}

*An asterisk indicates significant $(P \leq 0.05)$ differences between susceptible and resistant maize hybrids for individual harvest dates.

TABLE 2 | Aflatoxin accumulation in the developing maize kernels of resistant (Mp313E $\times$ Mp717) and a susceptible (GA209 × T173) hybrids inoculated with Aspergillus flavus in 2014 (21 days after pollination).

\begin{tabular}{lcc}
\hline Days after & \multicolumn{2}{c}{ Aflatoxin $(\boldsymbol{\mu g} / \mathbf{k g})$} \\
\cline { 2 - 3 } Inoculation & GA209 $\times$ T173 & Mp313E $\times$ Mp717 \\
\cline { 2 - 3 } 3 & $0 \pm 0$ & $0 \pm 0$ \\
4 & $0 \pm 0$ & $1 \pm 2$ \\
5 & $0 \pm 0$ & $2 \pm 3$ \\
6 & $0 \pm 0$ & $2 \pm 4$ \\
7 & $0 \pm 0$ & $2 \pm 3$ \\
8 & $18 \pm 9$ & $8 \pm 13$ \\
9 & $4 \pm 4$ & $2 \pm 3$ \\
10 & $10 \pm 17$ & $66 \pm 45$ \\
12 & $235 \pm 261^{*}$ & $643 \pm 706$ \\
14 & $2545 \pm 738$ & $0 \pm 0$ \\
16 & $248 \pm 394$ & $150 \pm 259$ \\
18 & $1856 \pm 1418$ & $578 \pm 382$ \\
20 & $4688 \pm 748^{*}$ & $146 \pm 218$ \\
22 & $1996 \pm 1212$ & $254 \pm 148$ \\
24 & $5745 \pm 1424^{*}$ & $507 \pm 717$ \\
\hline$A n$ & & \pm 07 \\
\hline
\end{tabular}

*An asterisk indicates significant $(P \leq 0.05)$ differences between susceptible and resistant maize hybrids for individual harvest dates.

Much higher levels of aflatoxin accumulation were found from 12 DAI through the last harvest 24 DAI in kernels of both hybrids. Significantly higher levels of aflatoxin contamination were found in the susceptible hybrid than in the resistant hybrid 20 and 24 DAI. Aflatoxin contamination was highest when the fungus would have invaded most of the endosperm and the scutellum as observed in the kernels processed in the histological part of this study.

\section{DISCUSSION}

Working with a weak pathogen such as A. flavus in pathological studies on developing maize kernels in the field is a challenge. 
Although a number of researchers have reported probable routes of A. flavus infection in developing kernels (Marsh and Payne, 1984a,b; Smart et al., 1990; Payne, 1992), it can be difficult to achieve adequate levels of $A$. flavus infection of kernels in field studies even when placing high numbers of conidia on external silks or inside ear husks (Windham et al., 2009). Thus, it was necessary for us to artificially inoculate developing kernels with A. flavus with our needle inoculator. In the field, maize ears are subjected to various environmental stresses (Odvody et al., 1997) and insect feeding (McMillian et al., 1980, 1985, 1990; Steffey et al., 1999; Windham et al., 1999) which damage the pericarp and provide A. flavus entry into the kernel. The needle inoculation method used in this study, in most cases, penetrated the seed pericarp and aleurone layer in the exposed crown end of the kernel similar to damage caused by insect probing and feeding.

In our study, the starting point of A. flavus infection of the inoculated kernels was in the wound through the pericarp, aleurone layer, into the endosperm. Researchers have found that A. flavus colonizes the endosperm to different degrees (Smart et al., 1990; Keller et al., 1994; Brown et al., 1995). In our study, the fungus readily colonized the starchy endosperm of the inoculated kernels. Our observations were similar to those of Dolezal et al. (2013) and Shu et al. (2015) although A. flavus colonized the endosperm at a slower rate in kernels of the resistant hybrid compared to the susceptible hybrid. Endosperm-specific antifungal proteins (PR-10, a 14-kDa trypsin inhibitor, and resistance-associate proteins) have been found in aflatoxin resistant germplasm and may explain the slower growth of A. flavus in kernels of the resistant hybrid (Chen et al., 2015).

Another route of kernel colonization by A. flavus is between the pericarp and aleurone layers. In our study, the fungus grew between these layers and formed a thickened hyphal mat within 5-7 DAI. Dolezal et al. (2013) observed minimal growth of A. flavus between the pericarp and aleurone layer toward the scutellum or germ 4 DAI. However, we found that A. flavus colonization between these layers was extensive and by $10-12$ DAI the fungus was beginning to colonize the germ and scutellum on the side opposite the endosperm via this route. Our findings agree with those of Brown et al. (1995) in which they found that the scutellum region could easily be colonized by $A$. flavus from the aleurone. One of the significant findings we observed was the extensive movement of $A$. flavus between the pericarp and aleurone. In a kernel of the susceptible hybrid collected 6 DAI, our inoculation with the needle did not penetrate the aleurone layer. The fungus had almost completely encircled the kernel between the pericarp and aleurone 6 DAI. The aleurone was still intact, and there was very minimal colonization of the endosperm or the scutellum. The intact aleurone served as a barrier limiting A. flavus colonization of the starchy endosperm and scutellum. In cereals, the aleurone layer is the outer layer of the endosperm and contains protein bodies and enzymes associated with endosperm digestion (Esau, 1977). The aleurone has also been found to contain antifungal proteins including a ribosome-inactivating protein (Guo et al., 1999).

Differences in colonization of the scutellum in kernels of the resistant and the susceptible hybrids were observed in our studies. In the susceptible hybrid, the scutellum was colonized at the endosperm-scutellum interface 4-5 DAI. In some cases, the A. flavus hyphae would mass outside the scutellum. Dolezal et al. (2013) and Shu et al. (2015) described the fungal mass as a biofilm which consisted of highly branched, tightly intertwined hyphae which pressed against the scutellum. This biofilm was not always necessary for infection of the scutellum, but was very common at the endosperm-scutellum interface. In our study, A. flavus also formed a similar structure at the endosperm-scutellum interface. Another significant event we observed, was the formation of an orderly mass of A. flavus hyphae at the endosperm-scutellum interface of resistant kernels 10 DAI. The fungal hyphae lined up at the surface of the scutellum of the resistant hybrid, but not in an extensive mass of hyphae similar to what we observed at the endospermscutellum interface in kernels of the susceptible hybrid. By this time point, A. flavus colonization of the scutellum in the susceptible hybrid was extensive. However, colonization of the scutellum in the resistant hybrid was limited to 2-4 cells deep. An organized layer of cells surrounding the scutellum appeared to be a barrier to fungal infection. One explanation of this defense mechanism could be that the glandular layer of the scutellum contains antifungal ribosome-inactivation proteins (Guo et al., 1999).

One route of kernel colonization by A. flavus we attempted to study was the infection of uninoculated kernels via the pedicel tip where the kernel is attached to the cob. Kernels are paired in their attachment to the cob in that the rachilla of the kernels is attached to the same vascular bundle (Smart et al., 1990). We inoculated all of the kernels surrounding select kernels to insure that the uninoculated kernel's paired kernel was infected with A. flavus. However, A. flavus was not found in any of the uninoculated kernels harvested 7, 14, and 21 DAI. Even with copious amounts of fungal hyphae and conidiophores located on adjacent kernels, none of these uninoculated kernels were infected with A. flavus. Smart et al. (1990) suggested that a point of A. flavus entry into developing kernel was the site of attachment to the cob. However, we did not observe any A. flavus colonization in the pedicel region of our paired, uninoculated kernels.

In maize breeding programs developing resistance to A. flavus, the accumulation of aflatoxin in developing ears in the field that have been artificially inoculated with this fungus is used to select resistant lines. In field evaluations, the level of $A$. flavus infection is lower in resistant lines compared to susceptible for lines. Since aflatoxin quantification is quicker and more efficient than determining fungal infection, levels of aflatoxin contamination are used by plant breeders and pathologists to identify resistant genotypes. Typically, inoculation techniques are used that cause limited kernel wounding. In our current study, we used a severe wounding technique to insure infection of kernels to be collected for histological purposes. This led to higher levels of aflatoxin contamination in the resistant lines than what we normally find in field evaluations. In our field evaluations, it not uncommon to find aflatoxin levels lower than $20 \mu \mathrm{g} / \mathrm{kg}$ in mature kernels of resistant lines (Williams et al., 2015; Brown et al., 2016).

Environmental conditions such as low rainfall and higher than normal ambient temperatures during kernel development can 
have a significant effect on aflatoxin accumulation (Windham et al., 2009). In our present study, mean ambient temperatures at time of inoculation in 2013 was $26^{\circ} \mathrm{C}$ and in 2014 was $22^{\circ} \mathrm{C}$. Initially, A. flavus colonization of kernel tissues was slowed in both hybrids in 2014. Optimum A. flavus growth has been reported at $29^{\circ} \mathrm{C}$ and optimum aflatoxin production by $A$. flavus was at $25^{\circ} \mathrm{C}$ (Schindler et al., 1967). As temperatures rose to normal levels during the 2014 experiment, high amounts of aflatoxin were found in the kernel tissues at the latter harvest dates. Aflatoxin contamination in maize field trials can fluctuate year to year as a result of temperature and soil moisture stresses on the plant (Widstrom, 1996; Windham and Williams, 1998, 2002).

Significant findings in our study were: (1) slower A. flavus internal colonization of kernels of the resistant hybrid compared to the susceptible hybrid, (2) A. flavus readily invaded the scutellum/germ tissue via mycelial growth between the pericarp and aleurone, and (3) the aleurone layer and the outer layer of the scutellum were found to be protective barriers limiting A. flavus colonization of the endosperm and scutellum/germ tissues.

\section{AUTHOR CONTRIBUTIONS}

GW conceived the study, made all inoculations, collected all kernel tissues, and oversaw processing of histological slides. WW

\section{REFERENCES}

Agbetiameh, D., Ortega-Beltran, A., Awuah, R. T., Atehnkeng, J., Cotty, P. J., and Bandyopadhyay, R. (2018). Prevalence of aflatoxin contamination in maize and groundnut in Ghana: population structure, distribution, and toxigenicity of the causal agents. Plant Dis. 102, 764-772. doi: 10.1094/PDIS-05-17-0749-RE

Anderson, H. W., Nehring, E. W., and Wichser, W. R. (1975). Aflatoxin contamination of corn in the field. Food Chem. 23, 775-782. doi: 10.1021/ jf60200a014

Ayeni, A. (2015). Extension education strategy for minimizing aflatoxin impact on sub-Saharan Africa agriculture and food systems. World Mycotoxin J. 8, 253-257. doi: 10.3920/WMJ2014.1716

Battilani, P., Toscano, P., Van der Fels-Klerx, H. J., Moretti, A., Camardo Leggieri, M., Brera, C., et al. (2016). Aflatoxin $B_{1}$ contamination in maize in Europe increases due to climate change. Sci. Rep. 6:24328. doi: 10.1038/ srep24328

Berlyn, G. P., and Miksche, J. P. (1976). Botanical Microtechnique and Cytochemistry. Ames, IA: Iowa State University Press.

Brooks, T. D., Williams, W. P., Windham, G. L., Willcox, M. C., and Abbas, H. K. (2005). Quantitative trait loci contributing resistance to aflatoxin accumulation in the maize inbred Mp313E. Crop Sci. 45, 171-174. doi: 10.1371/journal.pone. 0036892

Brown, R. L., Cleveland, T. E., Payne, G. A., Woloshuk, C. P., Campbell, K. W., and White, D. G. (1995). Determination of resistance to aflatoxin production in maize kernels and detection of fungal colonization using an Aspergillus flavus transformant expressing Escherichia coli $\beta$-glucuronidase. Phytopathology 85, 983-989. doi: 10.1094/Phyto-85-983

Brown, R. L., Williams, W. P., Windham, G. L., Menkir, A., and Chen, Z.-Y. (2016). Evaluation of African-bred maize germplasm lines for resistance to aflatoxin accumulation. Agronomy 6:24. doi: 10.3390/agronomy6020024

Castegnaro, M., and McGregor, D. (1998). Carcinogenic risk assessment of mycotoxins. Rev. Med. Vet. 149, 671-678.

Chen, Z.-Y., Rajasekaran, K., Brown, R. L., Sayler, R. J., and Bhatnagar, D. (2015). Discovery and confirmation of genes/proteins associated with maize aflatoxin resistance. World Mycotoxin J. 8, 211-224. doi: 10.3920/WMJ2014.1732 produced the seed in the field nursery, planted and maintained the plants, and made all of the pollinations of the test plants. JM, CR, and EW developed the techniques to analyze small kernel samples for aflatoxin and conducted all of the aflatoxin quantifications.

\section{FUNDING}

Funding for the research was provided by the United States Department of Agriculture's Agricultural Research Service.

\section{ACKNOWLEDGMENTS}

The authors express their appreciation to Ladonna Owens and Stewart G. Flint for the technical assistance in conducting the research. The authors also thank Amanda Lawrence of the Institute for Imaging and Analytical Technologies, Mississippi State University, for the preparation of all stained tissue sections. This paper is a joint contribution of the USDAARS and the Mississippi Agricultural and Forestry Experiment Station. Mention of trade names or commercial products in this publication is solely for the purpose of providing specific information and does not imply recommendation or endorsement by the USDA.

Darwish, W. S., Ikenaka, Y., Nakayama, S. M., and Ishizuka, M. (2014). An overview on mycotoxin contamination of foods in Africa. Toxicology 76, 789797. doi: 10.1292/jvms.13-0563

Dolezal, A. L., Obrian, G. R., Nielsen, D. M., Woloshuk, C. P., Boston, R. S., and Payne, G. A. (2013). Localization, morphology and transcriptional profile of Aspergillus flavus during seed colonization. Mol. Plant Pathol. 14, 898-909. doi: 10.1111/mpp.12056

Esau, K. (1977). Anatomy of Seed Plants, 2nd Edn. New York, NY: John Wiley and Sons.

Guo, B. Z., Cleveland, T. E., Brown, R. L., Widstrom, N. W., Lynch, R. E., and Russin, J. S. (1999). Distribution of antifungal proteins in maize kernel tissues using immunochemistry. J. Food Prot. 62, 295-299. doi: 10.4315/0362-028X62.3.295

Hawkins, L. K., Mylroie, J. E., Oliveira, D. A., Smith, J. S., Ozkan, S., Windham, G. L., et al. (2015). Characterization of the maize chitinase genes and their effect on Aspergillus flavus and aflatoxin accumulation resistance. PLoS One 10:e0126185. doi: 10.137/journal.pone.0126185

Keller, N. P., Butchko, R., Sarr, B., and Phillips, T. D. (1994). Visual pattern of mycotoxin production in maize kernels by Aspergillus spp. Phytopathology 84, 483-488. doi: 10.1094/Phyto-84-483

Lillehoj, E. B., Kwolek, W. F., Fennell, D. I., and Milburn, M. S. (1975). Aflatoxin incidence and association with bright greenish-yellow fluorescence and insect damage in a limited survey of freshly harvested high-moisture corn. Cereal Chem. 52, 403-411.

Liu, Y., and Wu, F. (2010). Global burden of aflatoxin-induced hepatocellular carcinoma: a risk assessment. Environ. Health Perspect. 118, 818-824. doi: 10.1289/ehp.0901388

Marsh, S., and Payne, G. A. (1984a). Scanning EM studies on the colonization of dent corn by Aspergillus flavus. Phytopathology 74, 557-561. doi: 10.1094/ Phyto-74-557

Marsh, S., and Payne, G. A. (1984b). Preharvest infection of corn silks and kernels by Aspergillus flavus. Phytopathology 74, 1284-1289.

McMillian, W. W., Widstrom, N. W., Wilson, D. M., and Evans, B. D. (1990). Annual contamination of Heliothis zea (Lepidoptera: Noctuidae) moths with Aspergillus flavus and incidence of aflatoxin contamination in preharvest corn 
in the Georgia coastal plain. J. Entomol. Sci. 25, 123-124. doi: 10.18474/07498004-25.1.123

McMillian, W. W., Widstrom, N. W., Wilson, D. M., and Hill, R. A. (1980). Transmission by maize weevils of Aspergillus flavus and its survival on selected corn hybrids. J. Econ. Entomol. 73, 793-794. doi: 10.1093/jee/73.6.793

McMillian, W. W., Wilson, D. M., and Widstrom, N. W. (1985). Aflatoxin contamination of preharvest corn in Georgia: a six-year study of insect damage and visible Aspergillus flavus. J. Environ. Qual. 14, 200-202. doi: 10.2134/ jeq1985.00472425001400020010x

Mehl, H. L., and Cotty, P. J. (2010). Variation in competitive ability among isolates of Aspergillus flavus from different vegetative compatibility groups during maize infection. Phytopathology 100, 150-159. doi: 10.1094/PHYTO-100-2-0150

Odvody, G. N., Spencer, N., and Remmers, J. (1997). A description of silk cut, a stress-related loss of kernel integrity in preharvest maize. Plant Dis. 81, 439-444. doi: 10.1094/PDIS.1997.81.5.439

Park, D. L., and Liang, B. (1993). Perspectives on aflatoxin control for human food and animal feed. Trends Food Sci. Technol. 4, 334-342. doi: 10.1016/09242244(93)90104-I

Payne, G. A. (1992). Aflatoxin in maize. Crit. Rev. Plant Sci. 10, 423-440. doi: 10.1080/07352689209382320

Reid, C. X., Sparks, D. L., Williams, W. P., and Brown, A. E. (2016). Single corn kernel aflatoxin $\mathrm{B}_{1}$ extraction and analysis method. Nat. Resour. 7, 405-410.

Schindler, A. F., Palmer, J. G., and Eisenberg, W. V. (1967). Aflatoxin production by Aspergillus flavus as related to various temperatures. Appl. Microbiol. 15, 1006-1009.

Scott, G. E., and Zummo, N. (1990). Registration of Mp313E parental line of maize. Crop Sci. 30:1378. doi: 10.2135/cropsci1990.0011183X003000060080x

Scott, G. E., and Zummo, N. (1992). Registration of Mp420 germplasm line of maize. Crop Sci. 32:1296. doi: 10.2135/cropsci1992.0011183X003200050054x

Shu, X., Livingston, D. P. III, Franks, R. G., Boston, R. S., Woloshuk, C. P., and Payne, G. A. (2015). Tissue-specific gene expression in maize seeds during colonization by Aspergillus flavus and Fusarium verticilliodes. Mol. Plant Pathol. 16, 662-674. doi: 10.1111/mpp.12224

Smart, M. G., Wicklow, D. T., and Caldwell, R. W. (1990). Pathogenesis in Aspergillus ear rot of maize: light microscopy of fungal spread from wounds. Phytopathology 80, 1287-1294. doi: 10.1094/Phyto-80-1287

Steffey, K. L., Rice, M. E., All, J., Andow, D. A., Gray, M. E., and Van Duyn, J. W. (eds) (1999). Handbook of corn insects. Lanham, MD: Entomological Society of America.

Tang, J. D., Perkins, A., Williams, W. P., and Warburton, M. L. (2015). Using genome-wide associations to identify metabolic pathways involved in maize aflatoxin accumulation resistance. BMC Genomics 16:673. doi: 10.1186/s12864015-1874-9

Wang, J. S., Huang, T., Su, J., Liang, F., Wei, Z., Liang, Y., et al. (2001). Hepatocellular carcinoma and aflatoxin exposure in Zhuqing Village, Fusui County, Peoples Republic of China. Cancer Epidemiol. Biomarkers Prev. 10, 143-146.

Warburton, M. L., Brook, T. D., Windham, G. L., and Williams, W. P. (2011). Identification of novel QTL contributing resistance to aflatoxin accumulation in maize. Mol. Breed. 27, 491-499. doi: 10.1007/s11032-010-9446-9
Warburton, M. L., Brooks, T. D., Krakowsky, M. D., Shan, X., Windham, G. L., and Williams, W. P. (2009). Identification and mapping of new sources of resistance to aflatoxin accumulation in maize. Crop Sci. 49, 1403-1408. doi: 10.2135/cropsci2008.12.0696

Widstrom, N. W. (1996). The aflatoxin problem with corn grain. Adv. Agron. 56, 219-279. doi: 10.1016/S0065-2113(08)60183-2

Wild, C. P., and Hall, A. J. (2000). Primary prevention of hepatocellular carcinoma in developing countries. Mutat. Res. 462, 381-393. doi: 10.1016/S1383-5742(00) 00027-2

Williams, W. P., Krakowsky, M. D., Scully, B. T., Brown, R. L., Warburton, M. L., and Windham, G. L. (2015). Identifying and developing maize germplasm with resistance to accumulation of aflatoxins. World Mycotoxin J. 8, 193-209. doi: 10.3920/WMJ2014.1751

Williams, W. P., and Windham, G. L. (2001). Registration of maize germplasm line Mp715. Crop Sci. 41, 1374-1375. doi: 10.2135/cropsci2001.4141374-ax

Williams, W. P., and Windham, G. L. (2006). Registration of maize germplasm line Mp717. Crop Sci. 46, 1407-1408. doi: 10.2135/cropsci2005.09-0330

Williams, W. P., and Windham, G. L. (2012). Registration of Mp718 and Mp719 germplasm lines of maize. J. Plant Regist. 6, 1-3. doi: 10.3198/jpr2011.09. $0489 \mathrm{crg}$

Windham, G. L., and Williams, W. P. (1998). Aspergillus flavus infection and aflatoxin accumulation in resistant and susceptible maize hybrids. Plant Dis. 82, 281-284. doi: 10.1371/journal.pone.0036892

Windham, G. L., and Williams, W. P. (2002). Evaluation of corn inbreds and advanced breeding lines for resistance to aflatoxin contamination in the field. Plant Dis. 86, 232-234. doi: 10.1094/PDIS.2002.86.3.232

Windham, G. L., Williams, W. P., and Davis, F. M. (1999). Effects of the southwestern corn borer on Aspergillus flavus kernel infection and aflatoxin accumulation in maize hybrids. Plant Dis. 83, 535-540. doi: 10.1094/PDIS.1999. 83.6.535

Windham, G. L., Williams, W. P., Hawkins, L. K., and Brooks, T. D. (2009). Effect of Aspergillus flavus inoculation methods and environmental conditions on aflatoxin accumulation in corn hybrids. Toxin Rev. 28, 70-78. doi: 10.1080/ 15569540802450037

Wu, F. (2015). Global impacts of aflatoxin in maize: trade and human health. World Mycotoxin J. 8, 137-142. doi: 10.3920/WMJ2014.1737

Conflict of Interest Statement: The authors declare that the research was conducted in the absence of any commercial or financial relationships that could be construed as a potential conflict of interest.

The reviewer EG-C and handling Editor declared their shared affiliation.

Copyright (c) 2018 Windham, Williams, Mylroie, Reid and Womack. This is an open-access article distributed under the terms of the Creative Commons Attribution License (CC BY). The use, distribution or reproduction in other forums is permitted, provided the original author(s) and the copyright owner are credited and that the original publication in this journal is cited, in accordance with accepted academic practice. No use, distribution or reproduction is permitted which does not comply with these terms. 